\title{
Zur Klinik der Folgezustände der Encephalitis lethargica.
}

\author{
Von \\ Dr. St. Danadschiieff, \\ Primarius der Abteilung für Nerven- und Geisteskrankheiten im Alexanderspital zu Sofia, Bulgarien.
}

(Eingegangen am 15. Februar 1920.)

Im Winter 1919/20 wurde auch Bulgarien von einer leichten Epidemie von Encephalitis lethargica heimgesucht. Die Zahl der vorgekommenen Fälle im ganzen Lande sind statistisch nicht anzugeben, da die Krankheit, als eine bis jetzt nicht beobachtete, weder den meisten Ärzten noch weniger der Bevölkerung bekannt war; trotzdem könnte man von einer Epidemie sprechen. Es sei bemerkt, daß zu gleicher Zeit auch zahlreiche Fälle von Influenza vorkamen, so daß diese beide Epidemien von Influenza und Encephalitis lethargica wenigstens jetzt concomitierende waren.

In den folgenden Zeilen werden wir im kurzen die Krankengeschichten von 13 von uns beobachteten Fällen von Encephalitis vorbringen, jedoch nicht aus der Periode der akuten Erscheinungen der Encephalitis, und zwar als die Kranken noch zu Bette lagen und von Temperatursteigerung, Somnolenz, Delirien, Paralyse der Augenmuskeln usw. befallen waren, sondern aus der Zeit, wo diese akuten Symptome längst vorbei waren und die Kranken angeblich von ihrer Schlafkrankheit genesen waren; bei allen unseren Kranken war eine Spanne Zeit von mindestens 2 und mehrere Monate verstrichen, nachdem sie das Bett verlassen haben und zu uns in Behandlung kamen.

Der Utbergang von dem akuten Stadium in den weiter unten zu schildernden subakuten oder chronischen Zustand von Encephalitis lethargica ist so unmerklich, daß weder die Kranke, noch die Angehörigen, selbst oft die Ärzte keinen Zusammenhang zwischen der überstandenen Schlafkrankheit und dem jetzigen Zustand fanden. Das hat uns veranlaßt, für diese Symptomenkomplexe, welche sehr wenig Gemeinsames mit der akuten Phase der Schlafkrankheit haben, die Bezeichnung „Folgezustände der Encephalitis lethargica“ zu wählen.

I. 41 jähr. Bauer erkrankte 2 Monate vor seinem Eintritt in das Spital (30. IV. 1920) an Fieber, Delirien, Nackensteifigkeit, Stechen im ganzen Körper. Mehr Einzelheiten kann er über seine Krankheit nicht angeben. Seit jener Zeit kann er nicht mehr arbeiten, und fühlt eine Eingenommenheit des Kopfes; ver- 
heiratet zum zweiten Mal; hatte von der ersten Frau 5 Kinder, - die zweite hatte 3 Totgeburten.

Mittelgroßer Mann; innere Organe ohne nennenswerte Veränderungen. Seitens des Nervensystems konstatierte man folgendes: Ungleiche Pupillen: $r .>1$, reagierend direkt und konsensuell auf Licht; aber die Reaktion auf Konvergenz und Akkommodation ist sehr schwach; leichte Ptosis auf $r$. Auge. Sonst die Augenbewegungen frei. Fundus oculi normal; die r. Nasolabialfalte ist schwächer ausgeprägt. Die Zunge und die Lippen zeigen leichte fibrilläre Zuchungen. Die Sprache zeigt keine Störungen, namentlich kein Silbenstolpern. Die mimische Muskulatur schwach beweglich. Hände und Körper zeigen leichten Tremor. Der Gang ist verlangsamt; die Körperhaltung leicht gebeugt. Die Haut- und Sehnenreflexe erhöht. Kein Romberg, kein Kernig, kein Babinsky, kein $\mathrm{Ny}$ stagmus. Keine psychischen Störungen.

Die subjektiven Beschwerden des Kranken bestehen in Eingenommenheit des Kopfes, Schwindelgefühl, Ohrensausen, Gefühl von Rigidität in den Halsmuskeln.

Zu bemerken ist, daß die Ungleichheit der Pupillen im Laufe der Zeit verschwand. Nach 2 monatlicher Behandlung wurde Pat. mit bedeutender Besserung entlassen.

2. 27 jähr. türkischer Leutnant erzählt, daß er vor 6 Monaten während 1 Monat an einer Krankheit, welche mit Temperatursteigerung und ununterbrochenem Schlaf verbunden war, litt; darauf folgte Schlaflosigkeit, die auf keine hypnotischen Mittel abweicht; jetzt klagt er über beständige Kopfschmerzen, Schwere und Eingenommenheit des Kopfes, welche sich bei der Bewegung steigern, leichte Dyspnöз und Beklemmungsgefühl.

Gut entwickelter Körperbau. Innere Organe normal, aber Herztätigkeit gesteigert. Puls 110-112. Pupillen gleich, weit, reagieren auf Licht, aber schwach auf Akkommodation und Konvergenz. Mimik unbeweglich, maskenartiger Gesichtsausdruck. Kein Nystagmus. Sprache langsam, aber ohne Silbenstolpern. Stimme schwach und monoton. Körperhaltung rigide wie bei Paralysis agitans; Gang langsam; der Kopf wird leicht nach links geneigt gehalten. Die Bewegungen der oberen Extremitäten sind ebenfalls langsam und zeigen bedeutende Rigidität; so z. B. wenn er beim Essen den Löffel oder den Bissen zu dem Munde bringt, so bleibt die Hand auf dem Wege eine Weile stehen und der Kranke verharrt in dieser kataleptischen Stel'ung wie vergeßlich auf die beabsichtigte Bewegung; Auf diese Weise dauert die Nahrungzaufnahme bei dem Kranken sehr lange Zeit. Das Bewußtsein ist erhalten, und außer einer leichten psychischen Depression, merkt man bei ihm keine psychischen Störungen.

Wassermann im Blute gab starke positive Reaktion, trotzdem der Kranke kategorisch jedwede luetische Infektion negiert.

Nach einer Beobachtung von 10 Tagen, wobei keine Veränderung in dem Krankhcitszustand zu bemerken war, reiste der Kranke nach seiner Heimat ab, und auf diese Weise entzog er sich der weiteren Beobachtung.

3. 40 jähr. Lehrer, aus gesunder Familie stammend, nie luetisch infiziert gewesen; erkrankte am 23. II. 1920 zuerst an Stechen im ganzen Körper und Schlaflosigkcit, welche nach mehreren Tagen vergingen. Im März machte er während 14 Tagen die ,Schlafkrankheit" durch: war bewußtlos, delirier te, hatte $38^{\circ}$ Temperatur. Wie er zu sich kam, bemerkte er Parese der 1. Gesichtshälfte, fühlte überall Muskelschmerzen, und als er zu gehen begann, merkte er, daß das linke Bein sich in Hüft- und Kniegelenke kontrahiere und sein Gang war somit sehr gestört.

Robuster Mann, innere Organe vollständig normal. 
Pupillen gleich, reagieren direkt und konsensuell auf Licht, aber sehr schwach auf Akkommodation und Konvergenz. Parese der beiden unteren Äste des 1 . Facialis; seitens der anderen Kopfnerven nichts Besonderes; Haut- und Sehnenreflexe lebhaft. Kein Kernig und kein Babinsky, kein Romberg. Keine Muskelschwäche. Beim Gehen bemerkt man folgendes: beim Heben des linken Beines um aufzutreten, es tritt in die hinteren Gruppen der Ober- und Unterschenkelmuskulatur, d. h. in die Flexoren ein tonischer Spasmus ein, infolgedessen wird das Bein in Hüft- und Kniegelenk flektiert und der Fuß erhält eine Pes-equinusStellung; der Kranke muß dabei einen Moment warten, bis der Spasmus etwas nachläßt, um ihn zu überwinden und auftreten zu können. Auf diese Weise wird der Gang des Patienten in höchstem Grade gestört. Zu gleicher Zeit, aber nicht immer, fühlt Pat. Schmerzen in der Fossa poplitea. Wassermann im Blute negativ.

Nach 1 monatlicher Behandlung verließ der Kranke das Spital ohne Besserung seines Zustandes.

4. 3l jähr. Bauer erzählt, daß er anfangs März 1920 an Fieber und Kopfschmerzen erkrankte und trat in die innere Abteilung des Alexanderspitals, wo man bei ihm die Schlafkrankheit diagnostizierte. Nach 40 Tagen wurde er aus dem Spital entlassen und bis Mitte August blieb er zu Hause, ohne jedoch arbeiten zu können.

Gut entwickelter Körperbau; innere Organe normal. Pupillen gewöhnlich gleich, aber manchmal zeigen sie eine vorübergehende Ungleichheit r. $>1$, reagieren direkt und konsensuell auf Licht, jedoch sehr schwach auf Konvergenz .und Akkommodation. Visus und Fundus normal. Corneal-, Skleral-, Rachen- und Abdominalreflexe sind vorhanden, der letztere sehr lebhaft, er wird sogar hervorgerufen, wenn man den Cremasterreflex, welcher selbst fehlt, hervorrufen will. Patellarsehnenreflex gesteigert, Babinsky +; Kernig -; Romberg +; keine Ataxie.

Gang langsam; die Körperhaltung starr wie bei Paralysis agitans; mimische Muskulatur unbeweglich. Händedruck r. schwächer als 1., aber sonst kein Unterschied zwischen rechts und links. Keine Atrophie in den Muskeln. Die Sprache ist langsam aber ohne Silbenstolpern. Puls etwas beschleunigt 92. Keine psychischen Störungen. Stuhlgang.

Pat. klagt über allgemeine Schwäche, unregelmäßigen Schlaf, Appetit und

Nach 2 monatlicher Behandlung wurde Pat. gebessert entlassen.

5. 32 jähr. Beamter. Anamnese ohne Belang. Ende Februar 1920 erkrankte Pat. mit Fieber und 2 Wochen dauernder Schläfrigkeit; die Umgebung meinte, er sei psychisch krank. Überführt nach seinem Heimatsort, blieb er durch 2 Monate wie erstarrt, schweigsam, aber beim Anreden gab er immer richtige Antworten, schlief sehr wenig.

Im Spital aufgenommen, stellte er folgendes Krankheitsbild dar: gut entwickelter Körper, innere Organe, außer einer Beschleunigung der Herztätigkeit (Puls 92), normal.

Pupillen mittelweit, gleich, reagieren auf Licht direkt und konsensuell, aber nicht auf Akkommodation und Konvergenz. Visus und Fundus normal. Kein Nystagmus; die Bewegungen der Augen frei nach allen Richtungen, aber nicht ausgiebig. Sprache frei ohne Silbenstolpern; kein Skandieren, kein Intentionstremor; Haut- und Sehnenreflexe gesteigert. Kein Kernig, kein Babinsky, kein Romberg. Wassermann im Blute negativ.

Der Gang ist langsam, Körperhaltung starr, Gesichtsausdruck maskenartig im allgemeinen eine Bewegungsarmut. - Keine Muskelatrophie; keine psy. chischen Störungen. 
Pat. klagt über allgemeine Müdigkeit, er ermüdet selbst beim Sitzen; wenn er sich ins Bett niederlegt, muß er gewisse Anstrengung anwenden, um sich von der allgemeinen Starre der Muskeln zu befreien und sich zu beruhigen; er schlafe sehr schwer ein und der Schlaf ist sehr kurz. Keine Kopfschmerzen; Unfähigkeit zu geistigen Arbeiten.

Nach 2 monatlichem Aufenthalt in der Abteilung wurde Pat. als geheilt entlassen; auch die Akkommodationsparalyse war geschwunden.

6. 40 jähr. Bauer. Anamn ese ohne Belang. 40 Tage vor der Aufnahme im Spital erkrankte Pat. an beständiger Schläfrigkeit, an Diplopie, an Vergeßlichkeit, Eingenommenheit, Apathie; von Zeit zu Zeit Beklemmungsgefuhl.

Hochgewachsener Mann, von mäßig entwickelter Muskulatur und Panniculus adiposus. Innere Organe normal. Pupillen gleich, reagieren direkt und konsensuell auf Licht, aber schwach auf Akkommodation und Konvergenz. Patellarsehnenreflex gesteigert, die ïbrigen Haut- und Sehnenreflexe normal. Die Beweglichkeit und die Sensibiität ohne Veränderung; der Gesichtsausdruck schläfrig. Körpertemperatur normal. Der Kranke liegt die meiste Zeit im Bette apathisch, aber auf alle Fragen gibt er sonst richtige Antworten; ïber die Zeit ist er nicht genau orientiert. Außer dieser Apathie und Zeitdesorientiertheit zeigt er keine anderen psychischen Störungen. Er klagte über Schläfrigkeit, ủber Kopfschmerzen und Eingenommenheit des Kopfes, über allgemeine Schwäche und Vergeßlichkit; der Schlaf war ruhig.

Aufgenommen am 14. IV. 1920. Der Kranke wurde am 20. V. gebessert entlassen.

7. 28 jähr. Oberleutnant, erzählt, daß er im vorigen Jahre in der Gefangenschaft an Malaria litt. Mitte März 1920 machte er im Verlauf von 10 Tagen eine Krankheit mit folgenden Symptomen durch: Fieber mit $38^{\circ} \mathrm{T}^{\circ}$, Schläfrigkeit, Doppelsehen, Delirien. Seit 1 Monat begann die Besserung, hat keine Diplopie mehr. Nicht luetisch gewesen.

Pupillen ungleich: r. $<1$, reagieren direkt und konsensuell auf Licht, aber nicht auf Akkommodation und Konvergenz. Keine anderen Storungen in den Augenmuskeln. Die Reflexe sind lebhaft. Beim ruhigen Sitzen merkt man leichtes Zittern des Kopfes und der Extremitäten, ebenso Zittern der Zunge; beim Aufreehtstehen und beim Gehen hört das Zittern auf. Die mimische Muskulatur wenig beweglich. Puls beschleunigt (112). Innere Organe und ihre Funktionen normal. Keine Schläfrigkeit, keine Kopfschmerzen.

Der Kranke wurde nur einmal von uns gesehen.

8. 20 jähr. Mann, erkrankte Ende Januar 1920 an Encephalitis lethargica: hatte während eines Monats Fieber, Somnolenz, Delirien; seit 11/2 Monaten hat er diese Symptome nicht mehr, statt deren aber fühlt er Eingenommenheit des Kopfes, leichte Ermudbarkeit, Rigidität („Zusammenschnüren“) im ganzen Körper.

Pupillen gleich, reagieren direkt und konsensuell auf Licht, aber nicht auf Akkommodation und Konvergenz. Patellarsehnenreflex erhöht; die übrigen Reflexe normal. Wegen der Rigidität der Muskeln sind die Bewegungen langsam. sonst keine körperlichen und psychischen Störungen.

9. 21 jähr. Hirt, erkrankte vor 9 Monaten als Soldat an der Schlafkrankheit: die ersten 15 Tage hatte er Fieber, Somnolenz und Delirien; als er zu sich kam, bemerkte er, daß er rechterseits hemiplegisch war und daß er ohne Beschwerden nicht sprechen kann. Aufgenommen in der Abteilung am 17. XI. 1920. Keine venerische Infektion; kein Alkoholmißbrauch. 
Gut entwickelter Körperbau. Innere Organe normal. Pupillen mittelweit, gleich, reagieren direkt und konsensuell auf Licht, aber nicht auf Akkommodation und Konvergenz. Leichtes Herabhängen des oberen Lides rechts. Die Reflexe lebhaft; die Patellarsehnenreflexe sehr erhöht. Babinsky fehlt. Mimische Muskulatur schwach beweglich. Die r. obere Extremität ist an Muskelkraft gleich der linken, aber man bemerkt in ihr gewisse Muskelrigidität, welche sich bei aktiven Bewegungen steigert, und eine Inkoordination bei der Ausführung von feineren Bewegungen. Bei den Bewegungen - tremor intentionis. Die untere Extremität zeigt ebenfalls Verminderung der motorischen Kraft. Beim Gehen jedoch zeigt das rechte Bein nicht jene charakteristische Haltung für die Hemiplegie, sondern der Fuß wird aufgehoben und bekommt eine Pes equinus-Stellung bis Pat. mit ihm auftritt; als Mitbewegung wird dabei die rechte obere Extremität hinaufgehoben und im Ellbogengelenk gebeugt gehalten; dieses Heben des Armes macht der Kranke, nach seiner Meinung, um das Gleichgewicht beim Gehen zu halten; wird der Arm aktiv oder passiv hinuntergelassen, so kann wieder der Kranke gehen, aber mit sichtlich beschwerlicherem Gang. Die mimische Muskulatur ist sehr schwach beweglich; die Sprache ist verlangsamt. Wassermann im Blute negativ.

Diese eigenartige Hemiplegie ist ein Folgezustand weder einer Embolie (das Herz ist normal), noch einer Trombose (Pat. war nicht erschöpft und marastisch während seines 15 tägigen Fieberzustandes), noch einer Hämorrhagie (junger 21 jähr. Mann, ohne Arteriosklerose, noch endlich einer Lues (keine luetische Infektion, Wassermann -), sondern sie ist das Resultat der pathologischen Veränderungen, welche die Encephalitis lethargica in den extrapyramidalen motorischen Zentren verursacht hat.

10. 22 jähr. Russe, gewesener Militärgefangener, erzählt, daß er als Bediensteter im Philipopeler Spital mit Fieber, Kopfschmerzen und beständiger Schläfrigkeit erkrankte. Wie er etwas gebessert war, wurde er transferiert und am 3. IV. 1920 in meine Abteilung aufgenommen. Behauptet keine luetische Infektion gehabt zu haben.

Körperlich gut entwickelter Mann. Innere Organe normal. Pupillen mittelweit, reagieren auf Licht direkt und konsensuell, aber nicht auf Akkommodation und Konvergenz. Kein Nystagmus oder Veränderungen in den übrigen Hirnnerven. Die Reflexe gesteigert. Kein Kernig und Babinsky. Mimische Muskulatur schwach beweglich. Sprache leicht dysartrisch beim Aussprechen von schwierigen Worten; Zungen- und Lippenzittern, Tremor des Kopfes; beim Schreiben zittert auch die Hand, aber die Schrift ist leserlich, ohne Auslassen von Silben oder Buchstaben. Wassermann im Blute negativ.

In psychischer Beziehung zeigt Pat. im allgemeinen eine Apathie und Sorglosigkeit für sein Schicksal, trotzdem er von Zeit zu Zeit beim Befragen den Wunsch äußert, nach der Heimat zu fahren; für die Zeit ist er nicht gut orientiert; ist leicht reizbar und reagiert zügellos auf kleine Reizungen. Oft zeigt er Anfälle von gehobener Stimmung, wobei er sehr hübsche russische Lieder singt.

Im Verlaufe von 8 monatlicher Beobachtung bis jetzt, merlst man eine Verminderung der ursprünglichen Apathie; er wurde lebhafter und beweglicher, äußert mehr Interesse für sich; die Akkommodationsparalyse jedoch, ebenso die schwache Beweglichkeit des mimischen Muskulatur, wie die erschwerte Sprache bestehen unverändert.

11. 13 jahr. Knabe erzahlt, daß er vor $7-8$ Monaten an Influenza, an welcher alle zu Hause litten, erkrankte; seine Krankheit dauerte 20 Tage und 15 Tage darauf erkrankte er an Schlafkrankheit, welche 15-16 Tage dauerte; dabei hatte 
er außer der Schläfrigkeit, Fieber und Kopfschmerzen. Seit dieser Zeit leidet er an Schlaflosigkeit.

Dem Alter entsprechend gut entwickeltes Kind. Innere Organe normal. Pupillen gleich, mittelweit, reagieren auf Licht, aber nicht auf Akkommodation und Konvergenz; z. B. beim Lesen werden die Pupillen nicht nur nicht eng, sondern weiter als beim ruhigen Sehen. Nystagmus -, Kernig - Babinsky - . Reflexe lebhaft. Mimik unbeweglich; Körperhaltung starr. Der Kranke nimmt unnatürliche Stellungen ein, der Kopf hängt nach unten, der Körper ist geneigt bald auf der einen, bald auf der anderen Seite, oder buckelförmig gebeugt; jedoch richtet sich der Kranke bei Befehl auf und kann sich durch gewisse Zeit gerade halten, aber allmählich nimmt er wieder die genannten katatonischen Stellungen ein. Den Mund hält er offen und der Speichel fließt heraus; aber bei Befehl verschluckt er ihn. In den Extremitäten merkt man außer der Rigidität auch Flexibilitas cerea. Der Gang ist langsam, die Körperhaltung dabei leicht gebeugt; wenn man aber den Kranken dazu anhält, kann er schneller gehen, selbst laufen. Die Stimme ist sehr schwach und monoton. Keine psychische Störungen; Schulkenntnisse erhalten. Der Kranke klagt über Schlaflosigkeit, allgemeine Schwäche und manchmal über Kopfschmerzen.

12. 15 jähr. Knabe, erkrankte vor 11 Monaten (Februar 1920) an Fieber, Somnolenz, Doppelsehen; die Krankheit dauerte 2 Wochen, aber die Schläfrigkeit, wenn auch in schwächerem Grade, besteht bis jetzt.

Leidlich gut entwickelter Knabe mit etwas blasser Haut und Schleimhäuten. Innere Organe normal. Pupillen mittelweit, gleich, reagieren direkt und konsensuell auf Licht, aber nicht auf Akkommodation und Konvergenz. Das Gesicht unbeweglich, die Haltung des Körpers und der Extremitäten starr. Der Mund steht leicht offen mit von Zeit zu Zeit ausfließendem Speichel; der Kranke kann aber bei Befehl den Mund zumachen und den Speichel verschlucken; ebenso kann er alle Befehle gut ausführen. Der Gang ist langsam, aber sicher. Nystagmus -, Kernig -, Babinsky - ; die übrigen Reflexe sind lebhaft. Die Patellarsehnenreflexe cher gesteigert. Hautsensibilität normal.

Der Kranke ist bei vollem Bewußtsein, aber von Zeit zu Zeit wird er, nach Angaben seiner Angehörigen, wie benommen und sehr reizbar; oft klagt er über Schläfrigkeit. Schulkenntnisse erhalten.

13. 14 jähr. Knabe erkrankte vor 8 Monaten an Fieber, 7 Tage und Nacht bestehende Somnolenz, Delirien, Diplopie, Kopfschmerzen; er war darauf durch 40 Tage in Spitalsbehandlung. Seit ca. 7 Monaten schäft er schwer ein und beim Erwachen fühlt er den Kopf schwer und benommen und den Körper im allgemeinen Zittern.

Dem Alter entsprechend gut entwickelter Knabe, zeigt keine Veränderungen der inneren Organe. Pupillen mittelweit, reagieren schwach auf Licht, gar nicht auf Akkommodation und Konvergenz; ja man bemerkt dabei gerade das Gegenteil, durch das Prävalieren des Dilatator pupillae, innerviert wie bekannt durch dem Sympathicus: läßt man z. B. den Kranken lesen, so werden die Pupillen statt eng sehr weit. Mimik unbeweglich; Körperhaltung starr, Kopf leicht geneigt, die Arme im Ellbogengelenk ziemlich gebeugt. Diese starre Korperhaltung ist morgens besonders stark ausgeprägt. Sonst sind keine Paresen in den Extremitäten: der Kranke bewegt sich ziemlich gut, trotz der rigiden Haltung; der Mund steht meist offen und der Speichel fließt oft aus dem Munde heraus; angehalten, den Speichel zu verschlucken, tut es der Pat. unverzüglich. Von Zeit zu Zeit wird Pat. von Zwangslachen befallen, die Reflexe sind gesteigert. Kein Nystagmus, kein Kernig, kein Babinsky. Keine psychischen Störungen. Die subjektiven 
Beschwerden bestehen in schwerem Einschlafen abends, Schläfrigkeit morgens, in Kreuzschmerzen, wenn er sich beugt.

Wie es aus den eben vorgestellten Krankheitsbildern leicht ersichtlich, haben wir hier nicht jene charakteristischen Symptome, bestehend vorwiegend aus Fieber, Somnolenz, Delirien, und auffallenden Paralysen der Hirnnerven-Symptome, welche das klinische Bild des Initialstadiums der Encephalitis ausmachen. Im Gegenteil, die Symptome, die hier auffallen, sind ganz andere; sie sind nämlich: die Parese oder die Paralyse der Akkommodationsmuskeln, die starre, der Paralysis agitans sehr ähnliche, Körperhaltung, die Unbeweglichkeit der mimischen Muskulatur, im allgemeinen die Bewegungsarmut (Strümpell), die Körper- und Nervenschwäche (Asthenie), die Schlaflosigkeit, der beschleunigte Puls, die extrapyramidalen Bewegungsstörungen. Dieser Symptomenkomplex, abgesehen von der seltener vorkommenden passageren Ungleichheit der Pupillen und den leichten psychischen Störungen, ist so prägnant und bildet ein so vollständiges klinisches Bild, fast grundverschieden von dem anfänglichen Bild der Encephalitis lethargica, daß es uns berechtigt erscheint, ihn als Syndrom der Folgezuständen der Encephalitis lethargica aufzustellen.

Die Akkommodationsparese und die Rigidität der Muskeln (der maskenartige Gesichtsausdruck ist eine Teilerscheinung dieser Rigidität) sind beständige Zeichen dieses Syndroms; wir möchten sie daher als pathognomonische bezeichnen.

Dieser Syndrom erinnert sehr an die Wilsonsche Krankheit, aber unterscheidet sich von der letzteren durch das Fehlen der braungrünlichen Pigmentierung des Hornhautrandes - spezifisches Symptom für diese Krankheit - und durch das Fehlen der Lebercyrrose. Er würde sich eher unter der zweiten Form des ,,amyostatischen Symptomenkomplexes" Strümpells, bestehend aus Starre, Bewegungsarmut, abnormen Dauerstellungen und Verharren der Glieder in der eingenommenen Lage, ohne Zittern, ohne Demenz, ohne Hornhautpigment subsummieren lassen; aber die Alkommodationsparese, welche in allen unseren Fällen bestand, unterscheidet deutlich unseren Syndrom von dem Strümpellschen.

Das Gemeinsame in der Wilsonschen Krankheit, im Strümpellschen amyostatischen und in unserem Syndrom der Folgezustände der Encephalitis lethargica ist das, daß es sich bei allen um eine Linsenkernaffektion handelt, aber während bei dem Wilsonschen und Strümpellschen Syndrom der Linsenkern infolge der Lebererkrankung, respektive direkt affiziert ist, bei unserem, wie wir meinen, er wird nachträglich durch Propagation des encephalitischen Prozesses von dem Augennervenkerne aus in Mitleidenschaft gezogen, was sich durch das früh- 
8 St. Danadschiieff: Zur Klinik der Folgezustände der Encephalitis lethargica.

zeitige Erscheinen und das gleichzeitige Bestehen der Akkommodationsparese bestätigt. Außerdem in allen unseren Fällen die Symptome die sie gegenwärtig zeigen, mit Ausnahme der Augenmuskellähmungen bestanden nicht zur Zeit des akuten Stadiums der Encephalitis, was ebenfalls beweist, daß sie sich nachträglich entwickelten.

Bemerkenswert bei unseren 13 Fällen ist, daß sie alle männliche Individuen betrafen. Nur zwei Frauen - der 14. und 15. Fall - haben wir je einmal gesehen, welche genau das gleiche Krankheitsbild darstellten, wie die übrigen. War das ein Zufall, oder sind die Männer im allgemeinen mehr empfänglich für die Encephalitis lethargica als die Frauen? Die meisten von unseren Kranken waren in mittlerem Alter, nur drei davon waren Knaben von 13 resp. 14 und 15 Jahren.

Was die Prognose der ,Folgezustände der Encephalitis" anbelangt, so müssen wir sie in bezug auf das Leben als ziemlich gute hinstellen; in unseren Fällen, solange wir sie beobachtet haben - und einen davon haben wir länger als 8 Monate unter den Augen -, hat sich weder eine Verschlimmerung, noch irgendwelche Komplikation, welche das Leben des Kranken etwa gefährdet hätten, entwickelt. Im Gegenteil haben wir in der Mehrzahl von ihnen eine größere oder kleinere Besserung gesehen; ja ein Fall war so weit gebessert, daß wir ihn als geheilt entlassen konnten; ein zweiter Kranker, nachdem er als gebessert entlassen wurde, erschien nach 5 Monaten zur Besichtigung, wobei er außer der Akkommodationsparese und der leichten Schwere im Kopfe keine andere Krankheitserscheinungen darbot; der Kranke konnte, nach seiner Aussage, mit leichter ländlicher Arbeit beschäftigt werden. Die Beobachtung über die meisten von unseren Kranken war nicht genügend lang, um über den endgültigen und dauernden Ausgang der Folgezustände der Encephalitis lethargica urteilen zu können. 